\title{
European dental graduate - moving back to the UK
}

\author{
Gulshan Dhanoya describes her positive experience of training in Europe \\ before returning to the UK - a process that was difficult, isolating and \\ frustratingly drawn out
}

W hen I packed my case to start a new chapter leaving home and starting my university life, unlike my school friends who were off to Leeds, Manchester, Bristol and Edinburgh, I was going that extra 1,000 miles, to study dentistry in Valencia, Spain.

My experience studying dentistry in Spain is something that is becoming more common, with higher university fees in the UK, high competition for places and more people wanting to experience life abroad.

I had the good fortune of learning a modern foreign language and making friends from all corners of the world, as well as studying a course I was passionate about. As my five years came to a close, I planned to return to the UK to start work.

I completed the situational judgement test and interview process for foundation training programmes in the UK, but despite being ranked in the top 200, I was not offered a training place.

Students who have completed dental school in the UK are offered priority, and I therefore embarked on the route of foundation equivalence training. Registering with the GDC in the UK is a fairly straightforward process, involving translating graduation certificates, academic transcripts and completing the necessary paperwork.

The wait for the GDC registration can be up to three months, and without this, you cannot apply for foundation equivalence.

The real complication is obtaining a performer number. This is coordinated between CAPITA and your local deanery. Foundation equivalence training requires the prospective student to find a trainer, who is more than four years qualified, to supervise, provide tutorials and mentor them over a period of time.
Each student hoping to carry out foundation equivalence training is subject to different 'conditions'. These are placed by the performers list decision group, based on the amount of experience the applicant has.

As a recently graduated dentist, I had little clinical experience, similar to all foundation dentists. This process of deciphering the clinical ability and hence the level of supervision required during the foundation equivalence training is a somewhat subjective process. I am aware that each deanery applies different rules and periods of time that supervision is required for.

In my case, the decision was that I would require ten months of supervision, and I was also sent a list of courses to attend. These included NHS rules and regulations, surgical extractions and an advanced restorative course. I also completed two audits, a PDP and a portfolio demonstrating my work. I was to attend these courses in my own time, tutorials were to be organised with my tutor in our own time, and no study days were provided by the deanery.

The move back from Europe was significantly harder than anticipated due to a variety of factors.

The administrative aspect with regards to registering with the GDC and applying for a performer number meant that I was waiting six months post-graduation before I could start working in the NHS.

The fact I was not on a training scheme was isolating, meaning I did not have the opportunity to get to know other young dentists at the same stage in their careers.

Having graduated from a European university, my undergraduate curriculum

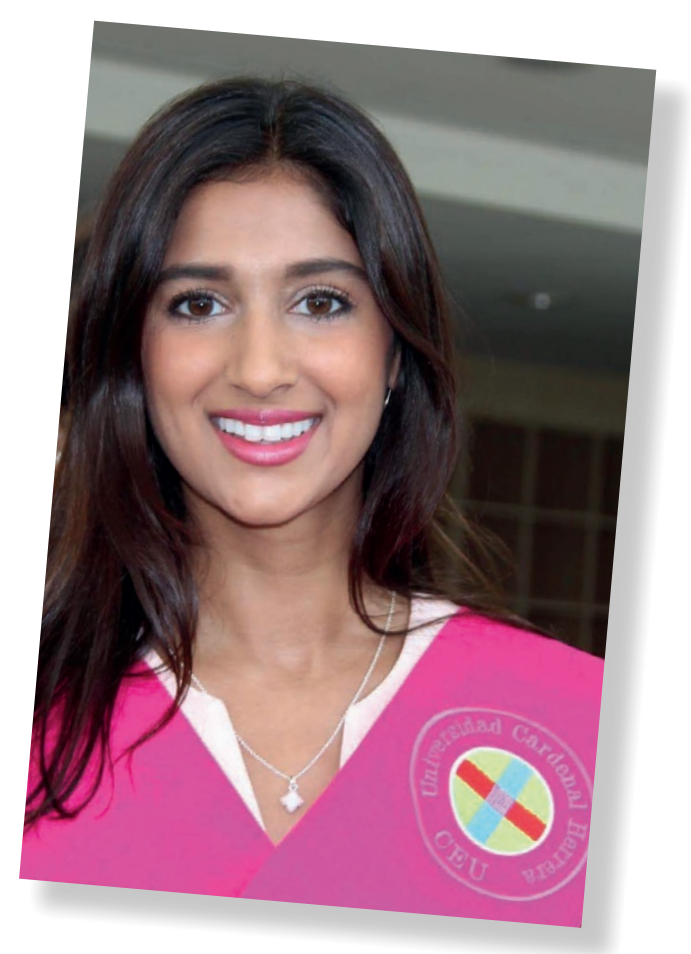

was not based on NICE guidelines or NHS rules and regulations. This coupled with no study days or guided syllabus in terms of foundation training, meant I faced added pressures that UK graduates did not.

I did find that undergraduate teaching in Spain taught me the full range of treatment options and was more focused on cosmetic dentistry. We did not ever use amalgam in clinics, so my ability to work with composite was good, and I had good experience with private crown and bridge preparations.

Having graduated more than two years ago, I now work full time with the Honour Health group and I have gone on to complete my MJDF examination, Invisalign training and many other short courses to improve my clinical skills.

Studying in Europe has so many benefits and I feel privileged to have had the opportunity to learn another language and culture. The move back to the UK is challenging in terms of losing touch with friends, starting your first job and at the same time trying to learn guidelines and regulations specific to the UK. Would I do it again? Yes!

With the current political climate and pending Brexit negotiations, I feel luckier than ever to have had the chance to live in Europe and return home to work. It isn't easy, but nothing worth having is, right? 